\title{
Synthesis and characterization of physicochemical and immunological properties of recombinant NS3-G2 dendrimer conjugate
}

\author{
Javadi $\mathbf{F}^{1}$, Rahimi $\mathbf{P}^{2 *}$, Modaressi $\mathbf{M H}^{1 \#}$, Shafiee Ardestani $\mathbf{M}^{3}$, Bolhassani $\mathrm{A}^{2}$, Aghasadeghi $\mathrm{MR}^{2}, \mathrm{Sadat} \mathrm{SM}^{2}$ \\ ${ }^{1}$ Department of Biology, Science and Research Branch, Islamic Azad University, Tehran, Iran. \\ ${ }^{2}$ Department of Hepatitis and AIDS, Pasteur Institute of Iran, Tehran, Iran. \\ ${ }^{3}$ Department of Radiopharmacy, Faculty of Pharmacy, Tehran University of Medical Sciences, Tehran, Iran.
}

\begin{abstract}
Introduction: An effective vaccine against HCV infection is not available. The non-structural protein 3 (NS3) of the virus as an important immunogenic candidate has been utilized in various modules. Nanostructured polymers have been recently used for efficient vaccine and drug delivery. The aim of the current study was the synthesis of rNS3-G2 conjugate and preliminary evaluation of its immunogenicity. Methods: The dendrimer was synthesized and conjugated with purified recombinant NS3 (rNS3) protein. The physicochemical properties of the conjugate were evaluated by Zeta potential, FT-IR spectra and confirmed by atomic force microscopy (AFM). Immunogenicity of the conjugate was assessed in BALB/c mice. Results: Synthesis and conjugation of dendrimer G2 with the protein were confirmed and immunological assays showed that the conjugated form of the antigen induced higher titer of IgG compared to rNS3 antigen alone. Conclusion: The results showed that the antigenic structure of rNS3 was maintained when conjugated with the biodegradable and biocompatible $\mathrm{G} 2$ dendrimers and the immunogenic properties of the antigen were enhanced. Therefore the new formulation may have potential as a vaccine candidate.
\end{abstract}

KEYWORDS: Adjuvant, Dendrimer G2, rNS3, HCV, Conjugate.

\section{INTRODUCTION}

Hepatitis $C$ virus (HCV) infection is a major public health concern with more than 170 million infected individuals worldwide of which approximately $70 \%$ remain infected for life [1]. Unfortunately, an effective vaccine against $\mathrm{HCV}$ is not available and although treatments with direct acting antivirals (DAAs) have shown a high rate of virological cure, the high costs and development of resistance make an effective therapeutic or prophylactic vaccine against the infection a necessity [2]. HCV is a positive-sense single strand RNA virus with approximately $9.6 \mathrm{kbp}$ genome, encoding a poly protein of 3000 amino acids long. The encoded poly protein is cleaved by viral and host proteases [3, 4]. The non-structural protein 3 (NS3) is bi-functional containing serine protease and RNA helicase activity and is directly involved in the virus replication $[5,6]$. The NS3 has been utilized as an important immune target in various vaccine and targeted antiviral therapy studies [7-11].

*Corresponding Author: Pooneh Rahimi, Department of Hepatitis and AIDS, Pasteur Institute of Iran, Tehran, Iran.

Email: pooneh5376@yahoo.com, prahimi@pasteur.ac.ir

Tel/Fax: (+98) 2166969291

\#Co-Corresponding Author: Mohammad Hossein Modarresi, Sciences and Research Branch, Islamic Azad UniversityTehran 1477893855, Iran.

Email: modaresi@tums.ac.ir

Tel/Fax: (+98) 2144865179
An effective vaccine for $\mathrm{HCV}$ infection should elicit strong cellular immune responses; however, subunit vaccines without a Th1-specific adjuvant formulation induce humoral responses, generally [12]. Several studies have shown that the use of particles in the nanoscale as carriers, in addition to stimulating humoral or cellular immunity, induces long-term immune responses [15-20]. Dendrimers are compact globular structures with advantageous properties including monodispersity, surface modification capabilities and the ability to control their sizes and molecular weights which make them ideal candidates to be used as carriers in the vaccine formulations $[17,21]$. The aim of the current study was to synthesize rNS3-G2 conjugate and to evaluate its immunogenicity in $\mathrm{BALB} / \mathrm{c}$ mice.

\section{MATERIALS and METHODS}

All the chemicals used were from Merck (Germany). The recombinant NS3 (rNS3) protein was prepared as previously described [22].

G2 dendrimer synthesis and conjugation with rNS3 protein

G2 dendrimer was synthesized as previously described [17]. Briefly, PEG600 was mixed with $3.72 \mathrm{mM}$ Dicyclohexylcarbodiimide (DCC) dissolved in Dimethyl 
sulfoxide (DMSO). Citric acid $(3.72 \mathrm{mM})$ was added to the mixture and the reaction was stopped by adding ddH2O. The solution was filtered through Watman filter paper (USA) and the filtrate was dialyzed for $16 \mathrm{~h}$ at room temperature against $\mathrm{ddH} 2 \mathrm{O}$. The dialyzed solution was freeze-dried and the G2 dendrimers $(1 \mathrm{mM})$ were mixed with $5 \mathrm{mg} / \mathrm{ml}$ of purified $\mathrm{rNS} 3$ in an EDC mediated reaction for $24 \mathrm{~h}$ at $4^{\circ} \mathrm{C}$. The conjugated NS3-G2 was purified by dialysis (Cutoff $10 \mathrm{kDa}$ ) and freezedried for further analysis.

\section{Characterization of the physicochemical properties of conjugated rNS3-G2}

FT-IR spectra were measured on a Bruker Model Tensor-27 spectrometer (Japan). To determine the size distribution and charge of the conjugated NS3, particle size and Zeta potential were measured using Malvern Nano-ZS (UK) at $24^{\circ} \mathrm{C}$ and water was used as a dispersant. Finally, AFM was used to study the structure and morphology of the conjugate.

\section{Immunization Protocols}

Pathogen-free, female BALB/c mice (weighting 18-20 g) were obtained from Pasteur Institute of Iran and handled according to the Pasteur Institute of Iran animal handling and care guidelines. Groups of seven mice were immunized subcutaneously (S.C.) in the tail base with $5 \mu \mathrm{g}$ of rNS3 antigen (rNS3-Ag) or the conjugated form of the antigen suspended in $100 \mu \mathrm{l}$ of PBS at weeks 0, 3 and 6. Mice blood samples were collected by retro-orbital bleeding two weeks post immunizations and the pooled sera were stored at $-70^{\circ} \mathrm{C}$ till used.

\section{Immunoassays by ELISA}

The antibody responses of the immunized mice (total IgG and $\mathrm{IgG}$ isotypes) were analyzed by ELISA [23]. Briefly, ELISA plates (Nunc, Denmark) were coated with purified recombinant
rNS3 $(3 \mu \mathrm{g} / \mathrm{ml})$ and incubated overnight at $4{ }^{\circ} \mathrm{C}$. After three times washing and blocking steps with PBS-T buffer, an optimum dilution of the pooled sera (1:1000 dilution for total IgG and 1:2000 for isotypes), was added to each well and incubated for $1 \mathrm{~h}$ at $37^{\circ} \mathrm{C}$, washed and further incubated with 1:10000 dilution of goat anti-mouse IgG-HRP conjugate (Sigma, USA) as secondary antibody. Finally, TMB (tetramethylbenzidine, Sigma, USA) was added and the absorbance was measured at $450 \mathrm{~nm}$. Dilutions of 1:2000 of goat anti-mouse IgG1, IgG2a, IgG2b and IgG3 antibodies (Sigma, USA) and anti-goat IgG-HRP conjugate $(1: 10000)$ were used respectively as primary and secondary antibodies for the $\mathrm{IgG}$ isotypes.

\section{Statistical analysis}

All experiments were performed in triplicate and SPSS software (SPSS, ver. 23) was used for the statistical analyses. Values less than $0.05(\mathrm{p}<0.05)$ were considered significant.

\section{RESULTS}

\section{Analysis of the rNS3-G2 conjugate}

FT-IR spectra showed that $1726 \mathrm{~cm}-1$ peaks of carbonyl groups of citric acid in the dendrimer structure were suppressed to $1651 \mathrm{~cm}-1$ in rNS3-G2 conjugate which is an indicative of the amide bond formation between $\mathrm{CO}$ group of the citric acid and NH group of the surface amino acids in the conjugate (Fig.1A). Furthermore, AFM imaging confirmed the morphology and particle surface level differences between the G2 dendrimers and rNS3-G2 conjugate. A uniform and non-turbulent state in the form of nanoparticles was also observed which indicated a successful conjugation between the two entities (Fig.1B).

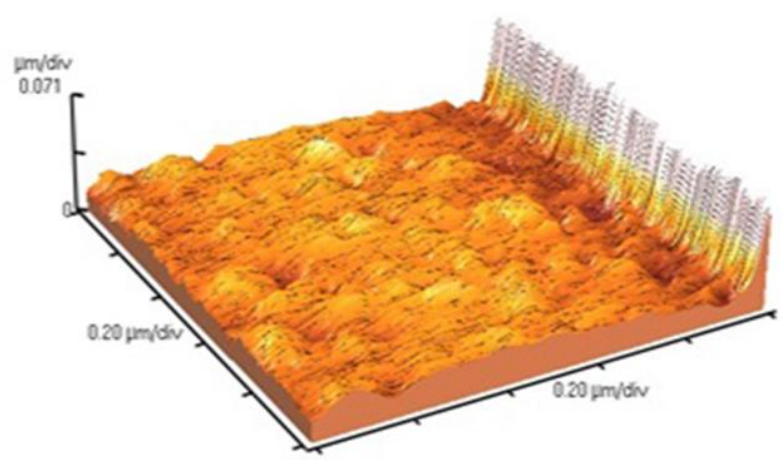

Non-conjugated G2

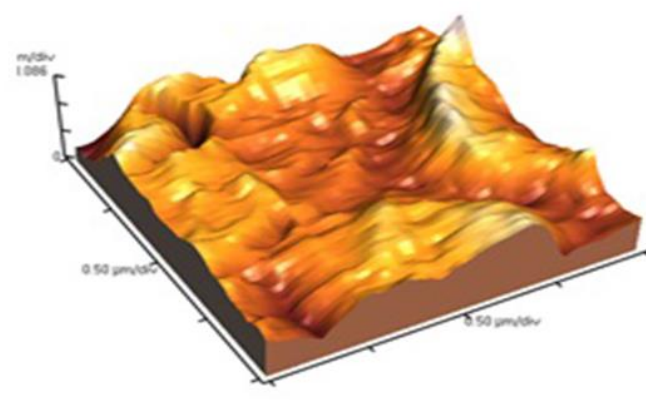

rNS3-G2 Conjugate

Fig.1. Physicochemical characterization of rNS3-G2 dendrimer conjugate. A) FT-IR spectra of G2 dendrimers in non-conjugated and conjugated forms with rNS3. B) AFM three-dimensional image of G2 dendrimers and rNS3-G2 conjugate. 
Mean zeta potentials were obtained as $-3.3 \mathrm{mV}$ and $3.12 \mathrm{mV}$ for G2 dendrimers and rNS3-G2, respectively. The size distributions were shown to be $90 \mathrm{~nm}$ and $215 \mathrm{~nm}$ for G2 dendrimers and rNS3-G2, respectively (Table1).

Table1. The charge and size distributions of the conjugated rNS3.

\begin{tabular}{|c|c|c|c|}
\hline Sample & Size $(\mathbf{n m})$ & Zeta Potential (mV) & PdI \\
\hline G2 & $90 \pm 4.5$ & $-3.3 \pm 0.5$ & 0.899 \\
\hline rNS3-G2 & $251.5 \pm 12$ & $3.12 \pm 0.5$ & 0.814 \\
\hline
\end{tabular}

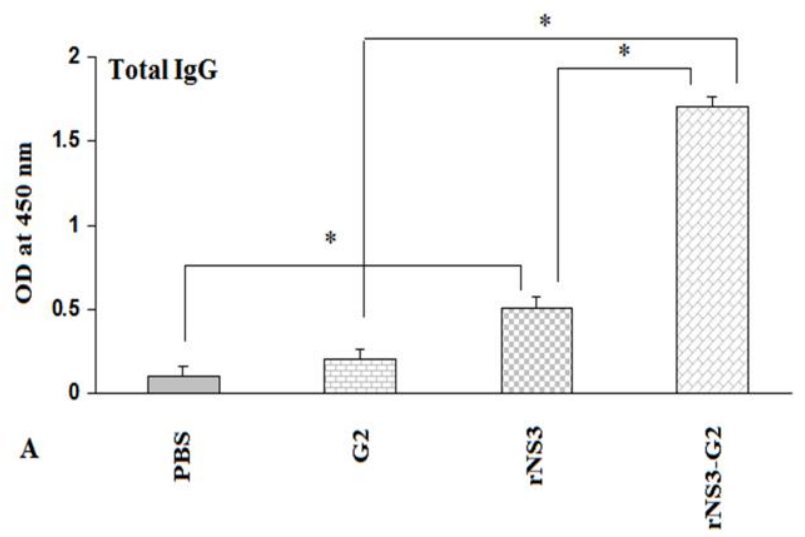

Evaluation of rNS3-Ag-specific antibody responses in mice

As shown in Fig. 2, no specific reactivity to the rNS3-Ag in sera of the negative control groups immunized by PBS or G2 dendrimers was detected. However, the groups vaccinated with either the purified antigen alone or rNS3-G2 induced significantly higher total IgG, compared to the control groups $(\mathrm{p}<0.05)$.

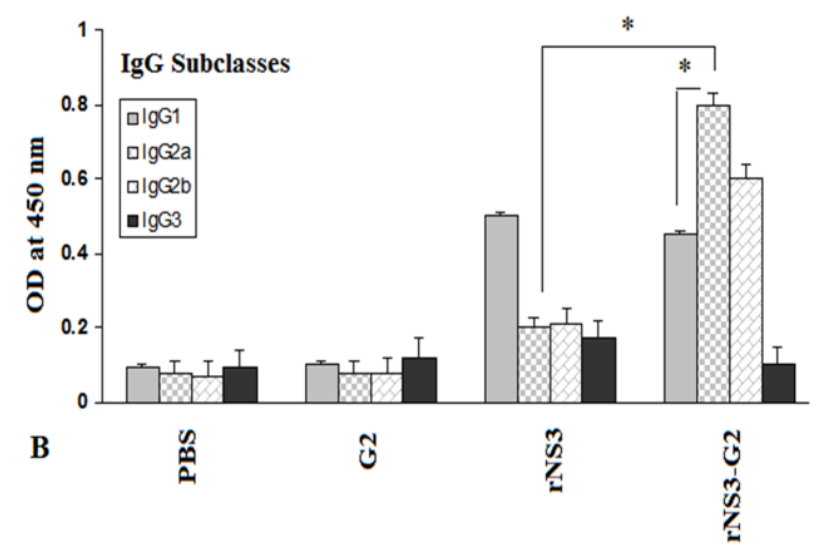

Fig. 2. Analysis of specific anti-NS3 antibody responses in mice immunized with rNS3-Ag and rNS3-G2 by ELISA. A) Total IgG. B) IgG subclasses in different immunized mice groups. Data represents antibody titers of pooled mice sera in three independent experiments and error bars indicate SD.

Furthermore, a statistically significant difference in total $\mathrm{IgG}$ response was observed in the group immunized with the conjugated protein compared to those that received rNS3 alone (Fig. 2A). The analysis of specific IgG isotypes indicated that IgG2a was the predominant subclass in the group immunized with rNS3-G2 (Fig. 2B).

\section{DISCUSSION}

Infection with $\mathrm{HCV}$ is a worldwide health concern and so far no promising vaccine against the infection is available [24]. Potent new direct acting antivirals (DAAs) have dramatically improved the success rates in disruption of the viral replication; however, high costs, side effects, and treatment failures still remain the main issues, underscoring the importance of developing therapeutic/prophylactic vaccines [25]. Several studies have shown that NS3 protein is a promising antigen for the purpose of anti-HCV vaccine development [26, 27]. However, subunit vaccines induce a lower immune response compared to whole cell vaccines, requiring improved vaccine carriers/adjuvants [28]. Dendrimer nanoparticles with a uniform small size (about $90 \mathrm{~nm}$ ) and many functional groups on the surface have been proposed as suitable carriers/adjuvants for presentation of subunit antigens [28]. The adjuvant effect of nanoparticles may include prolonged presentation, enhanced antigen uptake and direct stimulation of the innate immune system [29].

In this study, the G2 dendrimer nanoparticles were used as carriers and adjuvants and the immunogenicity of the conjugated protein was assessed in BALB/c mice. The size and Zeta potential of the rNS3-G2 compared to G2 alone showed that the conjugation reaction between the two components has occurred (Table 1). Moreover, AFM results further confirmed the conjugation between G2 and rNS3 (Fig.1). A suitable adjuvant can induce innate immune response, as well as specific cellular or humoral immune responses when formulated with a common antigen [30]. Currently, the only approved adjuvant for use in humans is an aluminum compound (i.e. alum) which leads to poor immune responses, requires multiple boosters and produces mainly a Th2- immune response [31]. Therefore, finding new adjuvants for use in humans is necessary. Our immunoassay analyses indicated that specificIgG titer in mice immunized with rNS3-G2 was significantly higher than rNS3 alone $(\mathrm{p}<0.009)$. Our result also showed that rNS3-G2 induces a Th1/Th2 mixed immunity with a higher level of IgG2a subtype production. In conclusion, our preliminary data exhibited that a new conjugated form of rNS3 protein with dendrimer G2, generated by chemical reactions, was capable of inducing higher immune responses compared to the non-conjugated antigen.

\section{ACKNOWLEDGEMENT}

The study was supported by Hepatitis and AIDS Department of Pasteur Institute of Iran (Grant No: 832) and was part of a Ph.D. program. We thank Dr. Salimi and Dr. Ahangari Kohan for their advice. These data was partially presented in 9th national biotechnology congress of Islamic Republic of Iran, 22- 23 May 2015 Tehran-Iran.

\section{CONFLICT OF INTEREST}

The authors declare that they have no conflict of interest.

\section{REFERENCES}

1. Larijani MS, Sadat SM, Nikbin M, Talebi SS, Javadi F, Mohajel N et al. Correlation Study Between IL-28B Gene Polymorphism (rs8099917SNP) 
and Sustained Virological Response in Iranian Patients with Chronic Hepatitis C. Clin Lab. 2016;62(3):417-23.

2. Majumdar A, Kitson MT, Roberts SK. Systematic review: current concepts and challenges for the direct-acting antiviral era in hepatitis C cirrhosis. Aliment Pharmacol Ther. 2016;43(12):1276-92. doi:10.1111/apt.13633.

3. Roohvand F, Kossari N. Advances in hepatitis $\mathrm{C}$ virus vaccines, Part one: Advances in basic knowledge for hepatitis $\mathrm{C}$ virus vaccine design. Expert Opin Ther Pat. 2011;21(12):1811-30. doi:10.1517/13543776.2011.630662. 4. Yazdanian M, Memarnejadian A, Mahdavi M, Sadat SM, Motevali F, Vahabpour R et al. Immunization of Mice by BCG Formulated HCV Core Protein Elicited Higher Th1-Oriented Responses Compared to PluronicF127 Copolymer. Hepat Mon. 2013;13(10):e14178. doi: 10.5812 /hepatmon. 14178 .

5. Moradpour D, Penin F. Hepatitis C virus proteins: from structure to function. Curr Top Microbiol Immunol. 2013;369:113-42. doi:10.1007/9783-642-27340-7_5.

6. Pouriayevali MH, Bamdad T, Aghasadeghi MR, Sadat SM, Sabahi F. Construction and Immunogenicity Analysis of Hepatitis C Virus (HCV) Truncated Non-Structural Protein 3 (NS3) Plasmid Vaccine. Jundishapur J Microbiol. 2016;9(3):e33909. doi:10.5812/jjm.33909.

7. BelonCA, Frick DN. Helicase inhibitors as specifically targeted antiviral therapy for hepatitis C. Future Virol. 2009;4:277-93.

8. Schlutter J. Therapeutics: new drugs hit the target. Nature. 2011;474(7350):S5-7. doi:10.1038/474S5a.

9. Furuta A, Salam KA, Akimitsu N, Tanaka J, Tani H, Yamashita A et al. Cholesterol sulfate as a potential inhibitor of hepatitis C virus NS3 helicase. J Enzyme Inhib Med Chem. 2014;29(2):223-9. doi: $10.3109 / 14756366.2013 .766607$.

10. Kiser JJ, Flexner C. Direct-acting antiviral agents for hepatitis C virus infection. Annu Rev Pharmacol Toxicol. 2013;53:427-49. doi:10.1146/annurev-pharmtox-011112-140254.

11. Rehman S, Ashfaq UA, Javed T. Antiviral drugs against hepatitis C virus. Genet Vaccines Ther. 2011;9:11. doi:10.1186/1479-0556-9-11.

12. Roohvand F, Kossari N. Advances in hepatitis C virus vaccines, part two: advances in hepatitis $\mathrm{C}$ virus vaccine formulations and modalities. Expert Opin Ther Pat. 2012;22(4):391-415. doi:10.1517/13543776.2012.673589.

13. Perrie Y, Mohammed AR, Kirby DJ, McNeil SE, Bramwell VW. Vaccine adjuvant systems: enhancing the efficacy of sub-unit protein antigens. Int J Pharm. 2008;364(2):272-80. doi:10.1016/j.ijpharm.2008.04.036.

14. Leroux-Roels G. Unmet needs in modern vaccinology: adjuvants to improve the immune response. Vaccine. 2010;28 Suppl 3:C25-36. doi:10.1016/j.vaccine.2010.07.021.

15. Jung T, Kamm W, Breitenbach A, Hungerer KD, Hundt E, Kissel T. Tetanus toxoid loaded nanoparticles from sulfobutylated poly(vinyl alcohol)-graft-poly(lactide-co-glycolide): evaluation of antibody response after oral and nasal application in mice. Pharm Res. 2001;18(3):352-60.

16. Kanchan V, Panda AK. Interactions of antigen-loaded polylactide particles with macrophages and their correlation with the immune response. Biomaterials. doi:10.1016/j.biomaterials.2007.08.015.
17. Khosravy MS, Shafiee Ardestani M, Ahangari Cohan R, Doroud D, Amini S, Momen SB. Et al. Design, Synthesis, Physicochemical and Immunological Characterization of Dendrimer-HBsAg Conjugate. VacRes. $2014 ; 1: 24-28$

18. Wendorf J, Chesko J, Kazzaz J, Ugozzoli M, Vajdy M, O'Hagan D et al. A comparison of anionic nanoparticles and microparticles as vaccine delivery systems. Hum Vaccin. 2008;4(1):44-9.

19. Xu Y, Tang H, Liu JH, Wang H, Liu Y. Evaluation of the adjuvant effect of silver nanoparticles both in vitro and in vivo. Toxicology letters. 2013; 219:42-8.

20. Asgary V, Kord Mafi O, Khosravy MS, Janani A, Namvar Asl N, Bashar R, et al. Evaluation of the effect of silver nanoparticles on induction of neutralizing antibodies against inactivated rabies virus. VacRes. $2014 ; 1: 33-34$

21. Ardestani MS, Fordoei AS, Abdoli A, Ahangari Cohan R, Bahramali G, Sadat SM et al. Nanosilver based anionic linear globular dendrimer with a special significant antiretroviral activity. J Mater Sci Mater Med. 2015;26(5):179. doi:10.1007/s10856-015-5510-7.

22. Javadi F, Rahimi P, Moham Modaressi MH, Bolhassani A, Shafiee Ardestani M, Aghasadeghi MR, et al. Evaluation of Truncated HCV-NS3 Protein for Potential Applications in Immunization and Diagnosis. Clin. Lab. 2016 ; 7: doi: 10.7754/Clin.Lab.2015.151118.

23. Sadat SM, Zabihollahi R, Aghasadeghi MR, Vahabpour R, Siadat SD, Memarnejadian A et al. Application of SCR priming VLP boosting as a novel vaccination strategy against HIV-1. Curr HIV Res. 2011;9(3):140-7.

24. Liang TJ. Current progress in development of hepatitis $C$ virus vaccines. Nat Med. 2013;19(7):869-78. doi:10.1038/nm.3183.

25. Sällberg M WM, Frelin L,. Therapeutic vaccines: challenges of chronic viral infections. Drug Discov Today Ther Strateg. 2006;4:253-66. 26. Takei S, Omoto C, Kitagawa K, Morishita N, Katayama T, Shigemura K et al. Oral administration of genetically modified Bifidobacterium displaying HCV-NS3 multi-epitope fusion protein could induce an $\mathrm{HCV}$ NS3-specific systemic immune response in mice. Vaccine. 2014;32(25):3066-74. doi:10.1016/j.vaccine.2014.03.022.

27. Qiu F, Bi S, Wang Y, Guo M, Yi Y, Chen S et al. Hepatitis C virusspecific cellular and humoral immune responses following immunization with a multi-epitope fusion protein. Int J Mol Med. 2012;29(1):12-7. doi:10.3892/ijmm.2011.801.

28. Vartak A, Sucheck SJ. Recent Advances in Subunit Vaccine Carriers. Vaccines (Basel). 2016;4(2). doi:10.3390/vaccines4020012.

29. Joshi VB, Geary SM, Salem AK. Biodegradable particles as vaccine antigen delivery systems for stimulating cellular immune responses. Hum Vaccin Immunother. 2013;9(12):2584-90. doi:10.4161/hv.26136.

30. Alavidjeh MS, Haririan I, Khorramizadeh MR, Ghane ZZ, Ardestani MS, Namazi H. Anionic linear-globular dendrimers: biocompatible hybrid materials with potential uses in nanomedicine. J Mater Sci Mater Med. 2010;21(4):1121-33. doi:10.1007/s10856-009-3978-8.

31. Marjiani DJ. Vaccine adjuvants: role and mechanisms of action in vaccine immunogenicity. Drug Discov Today. 2003;15;8(20):934-43.

32. Karla M, Limaa KM, dos Santos SA, Rodrigues JM. Vaccine adjuvant: it makes the difference. Vaccine 2004; 22: 2374-2379.

33. Lindblad EB. Aluminium adjuvants--in retrospect and prospect. Vaccine. 2004;22(27-28):3658-68. doi:10.1016/j.vaccine.2004.03.032. 